We agree that an 'adverse response' to gluten must be measured by changes in intestinal mucosal morphology. We are glad to be able to stress this, because an absence of symptoms cannot be equated with a lack of response to gluten.

We do not agree that gluten challenge is unnecessary in an infant with a flat biopsy who has not had acute enteritis, cows' milk intolerance, or IgA deficiency, and who responds to a gluten-free diet. Our clinical acumen is not as great as that of our Irish colleagues, and it is in such infants that we have difficulty in making a firm retrospective diagnosis of coeliac disease.

Lastly, the timing and technique of gluten challenge that Professor McNicholl and his colleagues use is only one of several apparently satisfactory regimens. We agree that there is the need for more precise definition of a positive response to gluten challenge, and that this need will remain until the 'cause' of coeliac disease is known.

A S McNeISH Department of Child Health, Clinical Sciences Building, Leicester Royal Infirmary, Leicester LE2 $7 L X$

H K HARMS Universitäts-Kinderklinik, Dr von Haunerschen Kinderspital, Munich, Western Germany

J REY

Hốpital des Enfants Malades, Paris, France

D H SHMERLING Kinderspital Steinwiesstrasse, Zurich, Switzerland

J K VISAKORPI Institute of Clinical Sciences, University of Tampere, Finland

J A WALKER-SMITH Department of Child Health, Queen Elizabeth Hospital for Children, London E2 8PS

\section{Technique for facilitating tendon reflexes in children}

Sir,

I do hope that Dr Varcasia's technique for eliciting tendon reflexes in a child by making him gag with a tongue depressor during examination of the throat ${ }^{1}$ will not become a common practice.

Any paediatrician who examines children will, one hopes, have learned at his mother's knees or will know instinctively that the most painful and distressing part of any examination should be left until last, and that no painful or distressing procedure should be used unless it can be justified. The picture of a child sobbing from this assault while the doctor attempts to obtain the errant reflex has elements both of tragedy and comedy. The suggestion that the assault be made by the mother seems particularly unpalatable, especially as this would appear to thwart the primary purpose of the throat inspection-to allow the doctor to see the throat and observe palatal movement.

One of the pleasures of working with children is that the doctor can allow himself to regress and to be a little childish. It is seldom difficult to obtain the co-operation of a child in reinforcing the reflexes by a variety of devices. Most children will happily squeeze their parent's finger, or even the doctor's, often taking an aggressive delight in doing so, and this will generally bring up a knee jerk which has been sluggish, if it is capable of being reinforced. A squeaky toy or rubber ball may be squeezed with the same effect, or the child can be persuaded to bite strongly on a sweet (jelly-babies are particularly useful since the child enjoys the symbolic make-believe).

Anxiety and tension will normally elicit tendon jerks, but many would consider the cost too high. An adequate neurological examination can be obtained in most children without upsetting them, or the doctor. This should surely be the aim to strive for.

\section{Reference \\ 1 Varcasia E. Letter: Technique for facilitating tendon reflexes in children. Arch Dis Child 1980; 55: 166. \\ EDWARD BRETT \\ Department of Neurology, The Hospital for Sick Children, Great Ormond Street, London WC1N $3 J H$}

Dr Varcasia comments:

I agree that no unjustified distressing procedure should be used and I also agree that distressing parts of the examination should be left until last, but I am unable to conceive as an assault any part of the examination of a child. I think that paediatricians must perform with gentleness any unpleasant manoeuvre that is necessary.

My technique for eliciting tendon reflexes is easy, rapid, and is nearly always successful; I use it only if other manoeuvres have failed or if I have not obtained the co-operation of the child. Consequently I think that my technique will not become common practice, but I do not see in it elements either of tragedy or comedy.

EUGENIO VARCASIA

Department of Paediatrics, General Hospital, 04100 Latina, Italy

\section{Lomotil in diarrhoeal illnesses}

Sir,

I was disturbed by the suggestion made by Dr Karan and Dr Limaye ${ }^{1-2}$ that Lomotil might have a place in the treatment of childhood diarrhoea in the tropics, and was 